\title{
Recurrence and Survival Outcomes After Cytoreductive Surgery and Hyperthermic Intraperitoneal Chemotherapy for Small Bowel Adenocarcinoma
}

\author{
AKSHAT SAXENA, SARAH J. VALLE, WINSTON LIAUW and DAVID L. MORRIS \\ University of New South Wales Department of Surgery, St. George Hospital, Kogarah, NSW, Australia
}

\begin{abstract}
Background: Peritoneal dissemination of small bowel adenocarcinoma (SBA) is rare but is associated with a dismal prognosis. Cytoreductive surgery and hyperthermic intraperitoneal chemotherapy (CRS-HIPEC) is a promising treatment option. We evaluated our experience of CRS-HIPEC for the treatment of SBA. Patients and Methods: Sixteen consecutive patients underwent CRS-HIPEC for small bowel malignancy between 2003 and 2016. Clinopathological and treatment-related factors were obtained from a prospective database. The study's endpoints of disease-free (DFS) and overall (OS) survival were evaluated using the Kaplan-Meier method. Prognostic variables were identified through univariate and multivariate analyses. Results: Follow-up was complete in all patients. The median follow-up was 20.6 (range=0.2-62) months. The was no in-hospital mortality and grade III/IV morbidity was $25 \%$. The median OS after CRS-HIPEC was 24.7 months, with a 36-month survival of $34 \%$. The median DFS was 11.3 months, with a 36-month DFS of 8\%. Two factors were associated with a poorer OS on univariate analysis; only peritoneal cancer index $>10$ was associated with a poorer OS on multivariate analysis ( $p=0.032)$. Conclusion: $C R S-H I P E C$ in selected patients with peritoneal dissemination of $S B A$ is associated with reasonable mid-term survival outcomes but treatment failure is common. High disease burden, quantified by the PCI is associated with poor outcomes. A large, prospective, multi-institutional study is needed to further evaluate the outcomes of CRS-HIPEC for SBA.
\end{abstract}

Small bowel malignancies are rare, accounting for only $1-3 \%$ of all gastrointestinal cancer $(1,2)$. Of these, small bowel adenocarcinoma (SBA) is the most common

Correspondence to: Akshat Saxena, UNSW Department of Surgery, St George Hospital, Kogarah, New South Wales, Australia. Tel: +61 29113 2070, Fax: +61 291133997, e-mail: akshat16187@gmail.com

Key Words: Small bowel adenocarcinoma, survival, recurrence, cytoreductive surgery, hyperthermic, intraperitoneal. histological subtype, accounting for approximately 40-60\% of all cases (1-3). The clinical presentation of SBA is often non-specific and characterized by a relatively asymptomatic early stage. Moreover, SBAs are difficult to identify at an early stage by most endoscopic or imaging modalities (4). Consequently, patients are often diagnosed at a late stage; up to $43 \%$ have metastatic disease on presentation $(5,6)$. These late presentations are associated with a median survival of only 8 to 20 months and a 5-year survival of $14-34 \%(7,8)$. Surgical extirpation of SBA is the only potentially curative treatment option (4).

The peritoneum is the most common site of metastasis from SBA. Although previously regarded as an incurable clinical entity, peritoneal dissemination in select patients with various malignancies, including colorectal cancer, appendiceal cancer and pseudomyxoma peritonei, has been successfully treated with the combined approach of cytoreductive surgery (CRS) and hyperthermic intraperitoneal chemotherapy (HIPEC) (9, 10). There are, however, limited data on the clinical efficacy of CRS-HIPEC for patients with SBA (11-14). The primary aim of this study was to evaluate the survival and recurrence outcomes of patients who underwent CRS-HIPEC for SBA in a high-volume center. As a secondary outcome, morbidity and prognostic factors were evaluated.

\section{Patients and Methods}

Patients. Approval number was not required because this study was deemed by the Institutional Review Committee to be of low to negligible risk given that it involves a retrospective analysis of data collected prospectively as part of the St George Peritoneal Surface Malignancy Program. All patients had consented for the provision of de-identified data as a part of this program. From November 2003 to January 2016, 16 consecutive patients with SBA underwent CRS and HIPEC by one surgical team for peritoneal carcinomatosis at St. George Hospital, Sydney, Australia. Selection of patients suitable for CRS-HIPEC was based on the extent of disease and ability to achieve a complete cytoreduction, performance status and comorbidities. The experimental protocols were approved by a Institutional Review Committee and meet the guidelines of the Australian government. 
Table I. Association of clinicopathological and treatment-related factors with overall survival (OS) and disease-free (DFS) survival on univariate analysis.

\begin{tabular}{|c|c|c|c|c|c|}
\hline Factor & $\begin{array}{c}\text { Number of } \\
\text { patients }\end{array}$ & $\begin{array}{l}\text { Median OS } \\
\text { (months) }\end{array}$ & $\begin{array}{c}p \text {-Value } \\
\text { (univariate) }\end{array}$ & $\begin{array}{c}\text { Median DFS } \\
\text { (months) }\end{array}$ & $\begin{array}{c}p \text {-Value } \\
\text { (univariate) }\end{array}$ \\
\hline Total & 16 & & & & \\
\hline Age at time of surgery & & & 0.15 & & 0.45 \\
\hline Mean \pm SD (range), years & $51.6 \pm 9.6(34-67)$ & & & & \\
\hline$<52$ Years, $\mathrm{n}$ & 10 & 21.9 & & 11.3 & \\
\hline$\geq 52$ Years, $\mathrm{n}$ & 6 & NR & & 8.3 & \\
\hline Gender, $\mathrm{n}$ & & & 0.40 & & 0.81 \\
\hline Male & 10 & 21.9 & & 11.3 & \\
\hline Female & 6 & 24.7 & & 10.2 & \\
\hline PCI & & & $0.004 * *$ & & 0.81 \\
\hline Mean \pm SD (range), $\mathrm{n}$ & $11.1 \pm 4.3(5-22)$ & & & & \\
\hline$<10$ & 9 & 45.1 & & 11.3 & \\
\hline$\geq 10$ & 7 & 23.1 & & 11.5 & \\
\hline Completeness of cytoreduction, $n$ & & & 0.12 & & 0.10 \\
\hline $\mathrm{CCO}$ & 15 & 24.7 & & 11.5 & \\
\hline $\mathrm{CC} 1 / 2$ & 1 & 18.1 & & 7.8 & \\
\hline Tumor differentiation, $\mathrm{n}$ & & & $0.04 * *$ & & 0.97 \\
\hline Good/moderate & 7 & 45.1 & & 11.3 & \\
\hline Poor & 4 & 18.1 & & 7.8 & \\
\hline Signet & 4 & 21.2 & & 8.3 & \\
\hline Time between diagnosis of primary and CRS-HIPEC & & & 0.34 & & 0.90 \\
\hline Mean \pm SD (range), months & $7.3 \pm 9.5(0.9-29.5)$ & & & & \\
\hline$<4$ Months, $\mathrm{n}$ & 9 & 24.7 & & 14.9 & \\
\hline$\geq 4$ Months, $\mathrm{n}$ & 7 & NR & & 10.2 & \\
\hline Primary location, $\mathrm{n}$ & & & 0.86 & & 0.12 \\
\hline Duodenum/jejunum & 11 & 34.0 & & 11.3 & \\
\hline Ileum & 5 & 21.9 & & 21.9 & \\
\hline Perineural invasion, $\mathrm{n}$ & & & 0.15 & & 0.81 \\
\hline Yes & 8 & 45.1 & & 11.3 & \\
\hline $\mathrm{N}$ & o & 4 & 21.2 & & 8.5 \\
\hline Unknown & 4 & & & & \\
\hline Lymphovascular invasion, $\mathrm{n}$ & & & 0.57 & & 0.11 \\
\hline Yes & 9 & 34.0 & & 14.9 & \\
\hline No & 3 & 21.9 & & 8.5 & \\
\hline Unknown & 4 & & & & \\
\hline Primary lymph node metastases, $\mathrm{n}$ & & & 0.61 & & 0.86 \\
\hline Yes & 9 & 34.0 & & 11.3 & \\
\hline No & 5 & 21.2 & & 10.2 & \\
\hline Unknown & 2 & & & & \\
\hline Preoperative chemotherapy, $\mathrm{n}$ & & & 0.08 & & 0.93 \\
\hline Yes & 4 & NR & & 10.2 & \\
\hline No & 12 & 21.1 & & 11.3 & \\
\hline Operation length & & & 0.61 & & 0.22 \\
\hline Mean \pm SD (range) & $7.7 \pm 2.2(3-12)$ & & & & \\
\hline$<8$ Hours, $\mathrm{n}$ & 7 & NR & & 10.2 & \\
\hline$\geq 8$ Hours, $\mathrm{n}$ & 9 & 24.7 & & 14.9 & \\
\hline $\mathrm{RBC}$ transfusion & & & 1.00 & & 0.80 \\
\hline Mean \pm SD (range) & $2.9 \pm 2.7(0-9)$ & & & & \\
\hline$<3, \mathrm{n}$ & 8 & 21.9 & & 10.2 & \\
\hline$\geq 3, \mathrm{n}$ & 8 & 24.7 & & 14.9 & \\
\hline EPIC, $n$ & & & 0.31 & & 0.062 \\
\hline Yes & 6 & 21.9 & & 8.5 & \\
\hline No & 9 & 24.7 & & 18.1 & \\
\hline Previous resection of primary tumor, $\mathrm{n}$ & & & 0.05 & & 0.42 \\
\hline Yes & 11 & 45.1 & & 11.5 & \\
\hline No & 5 & 18.1 & & 7.8 & \\
\hline
\end{tabular}


Table I. Continued

\begin{tabular}{|c|c|c|c|c|c|}
\hline Factor & $\begin{array}{c}\text { Number of } \\
\text { patients }\end{array}$ & $\begin{array}{c}\text { Median OS } \\
\text { (months) }\end{array}$ & $\begin{array}{c}p \text {-Value } \\
\text { (univariate) }\end{array}$ & $\begin{array}{c}\text { Median DFS } \\
\text { (months) }\end{array}$ & $\begin{array}{c}p \text {-Value } \\
\text { (univariate) }\end{array}$ \\
\hline Concomitant hepatic metastases, $\mathrm{n}$ & & & 0.27 & & 0.29 \\
\hline Yes & 3 & 20.0 & & 8.3 & \\
\hline No & 13 & 34.0 & & 11.5 & \\
\hline CEA & & & 0.75 & & 0.42 \\
\hline$<4 \mathrm{ng} / \mathrm{ml}$ & 8 & 34.0 & & 14.9 & \\
\hline$\geq 4 \mathrm{ng} / \mathrm{ml}$ & 7 & 21.9 & & 8.5 & \\
\hline Unknown & 1 & & & & \\
\hline CA19-9 & & & 0.74 & & 0.74 \\
\hline$<37 \mathrm{U} / \mathrm{ml}$ & 9 & 34.0 & & 11.5 & \\
\hline$\geq 37 \mathrm{U} / \mathrm{ml}$ & 6 & 24.7 & & 10.2 & \\
\hline Unknown & 1 & & & & \\
\hline CA125 & & & 0.64 & & 0.33 \\
\hline$<35 \mathrm{U} / \mathrm{ml}$ & 13 & 21.2 & & 10.2 & \\
\hline$\geq 35 \mathrm{U} / \mathrm{ml}$ & 2 & 21.9 & & 8.5 & \\
\hline Unknown & 1 & & & & \\
\hline Peritoneal carcinomatosis & & & 0.058 & & 0.36 \\
\hline Synchronous & 4 & 21.9 & & 10.2 & \\
\hline Metachronous & 12 & NR & & 11.3 & \\
\hline Number of peritonectomy procedures & & & 0.772 & & 0.25 \\
\hline Mean \pm SD (range) & $3.1 \pm 0.9(2-4)$ & & & & \\
\hline$<4, \mathrm{n}$ & 9 & 21.2 & & 11.3 & \\
\hline$\geq 4, \mathrm{n}$ & 7 & 21.9 & & 8.5 & \\
\hline
\end{tabular}

PCI: Peritoneal cancer index; CRS-HIPEC: cytoreductive surgery and hyperthermic intraperitoneal chemotherapy; RBC: red blood cell ; EPIC: early postoperative chemotherapy; CEA: carcinoembryonic antigen; CA19-9: carbohydrate antigen 19-9; CA125: carbohydrate antigen 125; SD: standard deviation.

Procedure. The extent of peritoneal disease was calculated and recorded using the peritoneal carcinomatosis index (PCI) as described by Jacquet and Sugarbaker (15). CRS was performed according to the Sugarbaker technique with the aim of removing all macroscopic peritoneal tumour (16). The completeness of cytoreduction was recorded as previously described (15). HIPEC was performed using the open-abdomen technique with mitomycin C $\left(12.5 \mathrm{mg} / \mathrm{m}^{2}\right.$ in $1.5 \%$ dextrose $)$ at $42.5^{\circ} \mathrm{C}$ for 90 minutes following CRS. Some patients underwent early postoperative intraperitoneal chemotherapy which was administered as $650 \mathrm{mg} / \mathrm{m}^{2}$ 5 -fluorouracil combined with $50 \mathrm{mEq}$ sodium bicarbonate in the immediate postoperative period for 5 consecutive days either in the intensive care unit (ICU) or high dependency unit.

Postoperative complications were graded according to the Clavien-Dindo Classification (17). Follow-up included clinical examination, measurement of tumour markers and review of thoracoabdominopelvic computed tomographic (CT) scans. All patients were followed-up monthly for the first 3 months after discharge and 6-monthly thereafter until the last time of contact or death. Recurrence was clinically defined as the return of tumour based on radiographic studies, presence of a palpable mass or elevated tumour markers. All patients suspected of recurrence were discussed amongst clinicians in a multi-disciplinary setting.

Statistical analysis. A prospectively maintained database was retrospectively analysed. All statistical analyses were performed using SPSS $^{\circledR}$ (Windows Version 22; IBM Corporation, New York, NY, USA).
Patient characteristics are reported using frequency and descriptive analyses. Comparison of normally distributed variables was performed using analysis of variance (one way-ANOVA) test. Categorical variables were analysed using the chi-square test or Fisher's exact test where appropriate. Hospital mortality was defined as death that occurred during the same admission for CRS-HIPEC. Multivariate analysis of prognostic factors for disease-free (DFS) and overall (OS) survival was performed using Cox regression. Median OS was calculated based on time between CRS-HIPEC and the last time of contact or death. Survival analysis was performed using the Kaplan-Meier method, which was measured from time of surgery, and tested for differences using the log-rank test. Significant difference was defined as $p<0.05$.

\section{Results}

Patient characteristics. Baseline characteristics are thoroughly summarized in Table I. There were 10 males $(63 \%)$. The mean age of patients was 51.6 \pm 9.6 (range $=34-$ $67)$ years. The location of the primary tumor was the duodenum in one $(6 \%)$ patient, jejunum in $10(63 \%)$ and ileum in five $(31 \%)$. Eleven $(69 \%)$ patients had their primary tumor resected prior to CRS-HIPEC. The mean length of time between diagnosis of primary and CRS-HIPEC was $7.3 \pm 9.5$ (range=0.9-29.5) months. Three (19\%) patients had concomitant hepatic metastases at the time of CRS-HIPEC. 
Table II. Summary of operative procedures performed.

\begin{tabular}{lc}
\hline Operative parameter & Number of patients \\
\hline Total & 16 \\
Anterior peritonectomy & 2 \\
Left upper quadrant peritonectomy & 0 \\
Right upper quadrant peritonectomy & 2 \\
Omentectomy & 14 \\
Pelvic peritonectomy & 13 \\
Lesser omentectomy & 0 \\
Partial hepatectomy & 3 \\
Partial gastrectomy & 1 \\
Small bowel resection & 12 \\
Hysterectomy & 1 \\
Colonic resection & 13 \\
Stoma formation & 8 \\
Cholecystectomy & 6 \\
Splenectomy & 3 \\
\hline
\end{tabular}

The mean PCI was 11 (range=5-22). Fifteen patients had a complete cytoreductive (CC0). Four (25\%) patients received preoperative systemic chemotherapy and 15 (94\%) adjuvant postoperative chemotherapy. Six (38\%) patients received EPIC. The mean number of peritonectomy procedures performed was $3.1 \pm 0.9$ (range $=2-4)$. A summary of the operative procedures that patients underwent is provided in Table II.

Adverse event outcomes. There was no in-hospital mortality. Morbidity grade was $0,1,2,3$ and 4 in two (13\%), two (13\%), eight (50\%), two (13\%) and two (13\%) patients, respectively. The most common complications were infection $(n=6,38 \%)$, pneumonia $(n=4,25 \%)$, intraabdominal collection $(n=4,25 \%)$, pleural effusion $(n=3$, $19 \%)$, fistula $(n=3,19 \%)$, postoperative ileus $(n=3,19 \%)$, and postoperative bleeding $(\mathrm{n}=2,13 \%)$. Two $(13 \%)$ patients needed re-operation for complications due to postoperative bleeding in one case and evacuation of hematoma in the other. The median length of intensive-care unit stay and overall hospital length of stay was 2 days (range $=1-8$ ) and 16 days (range $=7-41$ ), respectively.

Survival and recurrence outcomes. Follow-up was complete in all patients, with a median follow-up of 20.6 months (range $=0.2-62$ ). At the time of this study, $7(44 \%)$ patients were alive. The median OS of the whole-patient cohort was 24.7 months (Figure 1). The 12-, 36-, and 60- month survival rates were $93 \%, 34 \%$ and $17 \%$, respectively. Twelve (75\%) patients had developed recurrence by the time of this study. The median DFS was 11.3 months. The 12-, 24- and 36month DFS rates were $42 \%, 8 \%$ and $8 \%$ months, respectively.

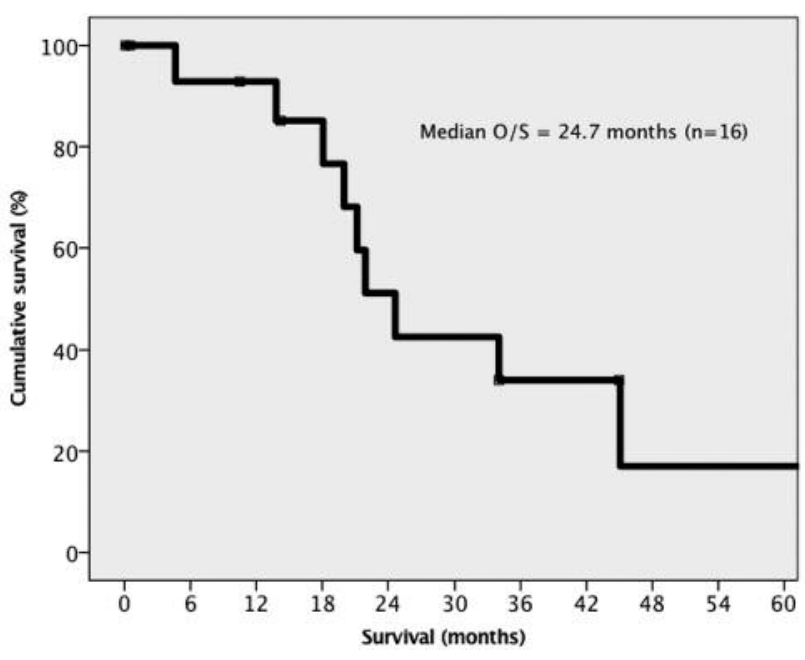

Figure 1. Overall survival after cytoreductive surgery and hyperthermic intraperitoneal chemotherapy.

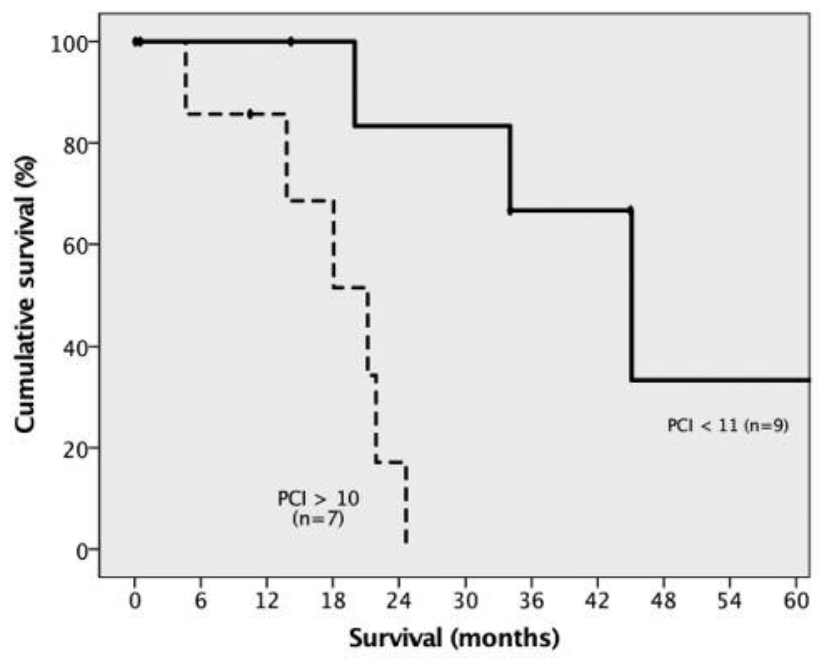

Figure 2. Overall survival stratified by peritoneal cancer index (PCI) $(p=0.032)$.

Prognostic factors for survival and recurrence. A tabulation of the prognostic impact of clinicopathological and treatmentrelated factors on OS and DFS is provided in Table I. Two factors were associated with a poorer OS on univariate analysis; $\mathrm{PCI}^{3} \geq 10$ (versus $<10, p=0.004$ ) (Figure 2) and poor tumour differentiation (signet versus poor versus good/moderate; $p=0.041$ ). Only PCI retained association with a poorer OS on multivariate analysis (hazard ratio $=10.99 ; 95 \%$ confidence interval=1.2-97.9; $p<0.032$ ). No factors were associated with a poorer DFS on univariate or multivariate analysis. 


\section{Discussion}

Our study demonstrates that CRS-HIPEC for select patients with SBA is safe and relatively efficacious. There was no inhospital mortality. Moreover, our grade III/IV morbidity rate of $25 \%$ is less than we have reported for other malignancies undergoing CRS-HIPEC $(18,19)$. This likely reflects the fact that at our Institution, like most others, CRS-HIPEC is generally reserved for patients with limited disease SBA who require a less extensive cytoreduction.

We report a median OS of 24.7 months with a 3-year survival rate of $34 \%$. Our results are commensurate with those from other series. Van Oudheusden and colleagues performed a multicentre Dutch study of 16 patients treated with CRS-HIPEC between 2005-2014 and showed a median survival of 30.8 months with a 3-year survival rate of $49 \%$ (14). In contrast, the three patients who had advanced disease and were unsuitable for complete cytoreduction had a median survival of only 7.1 months. Sun and colleagues evaluated 17 patients who underwent CRS-HIPEC for SBA and showed a median OS of 18.4 months; the 3-year survival rate was $23 \%$ (12). Liu and colleagues evaluated 31 patients from Japan with small bowel malignancy who were referred for CRS-HIPEC, of these, 25 had SBA. Of the 31 patients, 21 underwent CRS-HIPEC and the remaining 10 were treated conservatively. The authors reported that in those who underwent CRS-HIPEC, the median OS was 36 months, and the 3-year survival rate was $40 \%$. The delivery of HIPEC conferred a significant survival advantage $(p<0.001)(11)$. Elias and colleagues performed a multiinstitutional comparative study of 440 French patients who underwent complete CRS-HIPEC, of these 31 had a small bowel primary tumor. The number of patients with SBA was not specified. The median OS in this group was 47 months, with a 5 -year survival rate of $51 \%$ (20). Overall, a careful review of the literature shows that in select patients with SBA, CRS-HIPEC is associated with a median survival of 18-47 months, and with a 3-year survival rate of $23-51 \%$.

Despite promising survival outcomes, most patients undergoing CRS-HIPEC for SBA develop treatment failure. In our study, 12 (75\%) patients developed recurrent disease after a median follow-up of 20.6 months. The median DFS was 11 months, with a 2-year DFS rate of $8 \%$. Intra-abdominal treatment failure was most common (10 out of $12,83 \%)$; other sites of recurrence included the femur and liver in one $(6 \%)$ patient each, respectively. Similar results have been reported elsewhere. Van Oudheusden and colleagues reported a median DFS of only 9.5 months (14). Sun and colleagues did not report DFS data but of the 17 patients in their cohort, 16 developed recurrent disease during follow-up. These data highlight the importance of strict patient selection and the need to evaluate adjuvant therapies after CRS-HIPEC for SBA.

Although the prognosis after CRS-HIPEC in patients with
SBA is guarded, these data must not be interpreted in isolation. In particular, it must be noted that the natural history of metastatic SBA is dismal, with a median survival of 3-12 months. According to the United States National Cancer Data Base, the 5-year OS for patients with stage IV SBA was only $4 \%$ (21). Treatment with systemic chemotherapy and conventional surgery have also yielded poor outcomes. In 2005, the Eastern Cooperative Group reported a median OS of 8 months in 38 patients with SBA who were treated with 5-fluorouracil, doxorubicin and mitomycin C (22). Fishman and colleagues evaluated 44 patients who received palliative chemotherapy for metastatic or locally advanced SBA. Many different chemotherapy regimens with a variety of drugs were used. The median survival of patients who received first-line chemotherapy was 11 months, with an overall response rate of $36 \%$ (23). Overman and colleagues reviewed 80 patients with metastatic SBA who were treated at the M.D Anderson Cancer Centre between 1978 and 2005. The authors reported an overall response rate of $25 \%$ with a median OS of 13 months and median progression free survival of 4.6 months. The authors showed that patients who received 5-fluorouracil and a platinum agent had a longer DFS (8.7 months) than patients who received other chemotherapy combinations (3.9 months; $p=0.003)$. This did not, however, translate into a significant survival benefit (14.8vs. 12 months; $p=0.1$ ) (2). Overall, the prognostic benefit of systemic therapy for metastatic SBA is unclear but it may have a valuable role as an adjunct therapy to CRS-HIPEC for select patients.

Given the need to optimize patient outcomes and reduce the likelihood of futile treatment, it is important to apply strict selection criteria. Our study showed that the extent of disease, quantified by the PCI, is prognostic on multivariate analysis. Median OS in patients with a PCI $<10$ was 45 months compared to 23 months in those with a PCI of 10 or more. Liu and colleagues reported a median OS of only 12 months in patients with a PCI $>15$, whereas median OS was not reached in patients with a PCI $<15$ (11); the 5-year OS rates for the two groups were $0 \%$ and $65 \%$, respectively. Similar data has been published elsewhere (20). These data suggest that it may be prudent to restrict CRS-HIPEC to patients with a PCI $<15$ given the poor outcomes in patients with more extensive disease.

Our study has several limitations. Firstly, it was a retrospective analysis of prospectively collect data and was subject to the same limitations as other retrospective studies. Confounding by unknown variables may have reduced the validity of the analyses on prognostic variables. Secondly, the study had a relatively low number of patients. This, however, reflects the rare nature of SBA. Our Institution is a high-volume, quaternary referral institution which manages most cases of peritoneal carcinomatosis in Australia. The relative rarity of SBA has consistently precluded clinicians from collecting high quality clinical 
data which can then be used to evaluate treatment options and develop clinical guidelines. It also makes it difficult to perform randomized controlled trials; thus, a collaborative multicentre approach to collecting data and performing clinical trials is clearly indicated.

In conclusion, CRS-HIPEC for SBA is safe and relatively efficacious. Treatment failure, however, is common. Strict patient selection incorporating a thorough evaluation of disease extent may improve outcomes. The relative rarity of SBA also makes it essential for a collaborative approach to evaluate outcomes and the role for adjuvant therapies.

\section{Conflicts of Interest}

The Authors report no conflict of interest.

\section{References}

1 Weiss NS and Yang CP: Incidence of histologic types of cancer of the small intestine. J Natl Cancer Inst 78(4): 653-656, 1987.

2 Overman MJ, Kopetz S, Wen S, Hoff PM, Fogelman D, Morris J, Abbruzzese JL, Ajani JA and Wolff RA: Chemotherapy with 5 -fluorouracil and a platinum compound improves outcomes in metastatic small bowel adenocarcinoma. Cancer 113(8): 20382045, 2008.

3 Neugut AI, Jacobson JS, Suh S, Mukherjee R and Arber N: The epidemiology of cancer of the small bowel. Cancer Epidemiol Biomarkers Prev 7(3): 243-251, 1998.

4 Shenoy S: Primary small-bowel malignancy: Update in tumor biology, markers, and management strategies. J Gastrointest Cancer 45(4): 421-430, 2014.

5 Bauer RL, Palmer ML, Bauer AM, Nava HR and Douglass HO, Jr.: Adenocarcinoma of the small intestine: 21-year review of diagnosis, treatment, and prognosis. Ann Surg Oncol 1(3): 183-188, 1994.

6 Frost DB, Mercado PD and Tyrell JS: Small bowel cancer: A 30year review. Ann Surg Oncol 1(4): 290-295, 1994.

7 Garcia Marcilla JA, Sanchez Bueno F, Aguilar J and Parrilla Paricio P: Primary small bowel malignant tumors. Eur J Surg Oncol 20(6): 630-634, 1994.

8 Williamson RC, Welch CE and Malt RA: Adenocarcinoma and lymphoma of the small intestine. Distribution and etiologic associations. Ann Surg 197(2): 172-178, 1983.

9 Chua TC, Moran BJ, Sugarbaker PH, Levine EA, Glehen O, Gilly FN, Baratti D, Deraco M, Elias D, Sardi A, Liauw W, Yan TD, Barrios P, Gomez Portilla A, de Hingh IH, Ceelen WP, Pelz JO, Piso P, Gonzalez-Moreno S, Van Der Speeten K and Morris DL: Early- and long-term outcome data of patients with pseudomyxoma peritonei from appendiceal origin treated by a strategy of cytoreductive surgery and hyperthermic intraperitoneal chemotherapy. J Clin Oncol 30(20): 2449-2456, 2012.

10 Elias D, Goere D, Dumont F, Honore C, Dartigues P, Stoclin A, Malka D, Boige V and Ducreux M: Role of hyperthermic intraoperative peritoneal chemotherapy in the management of peritoneal metastases. Eur J Cancer 50(2): 332-340, 2014.
11 Liu Y, Ishibashi H, Takeshita K, Mizumoto A, Hirano M, Sako S, Takegawa S, Takao N, Ichinose M and Yonemura Y: Cytoreductive surgery and hyperthermic intraperitoneal chemotherapy for peritoneal dissemination from small bowel malignancy: Results from a single specialized center. Ann Surg Oncol 23(5): 1625-1631, 2016.

12 Sun Y, Shen P, Stewart JH, Russell GB and Levine EA: Cytoreductive surgery and hyperthermic intraperitoneal chemotherapy for peritoneal carcinomatosis from small bowel adenocarcinoma. Am Surg 79(6): 644-648, 2013.

13 Marchettini P and Sugarbaker PH: Mucinous adenocarcinoma of the small bowel with peritoneal seeding. Eur J Surg Oncol 28(1): 19-23, 2002.

14 van Oudheusden TR, Lemmens VE, Braam HJ, van Ramshorst B, Meijerink J, te Velde EA, Mehta AM, Verwaal VJ and de Hingh IH: Peritoneal metastases from small bowel cancer: Results of cytoreductive surgery and hyperthermic intraperitoneal chemotherapy in the netherlands. Surgery 157(6): 1023-1027, 2015.

15 Jacquet $\mathrm{P}$ and Sugarbaker PH: Clinical research methodologies in diagnosis and staging of patients with peritoneal carcinomatosis. Cancer Treat Res 82: 359-374, 1996.

16 Sugarbaker PH: Peritonectomy procedures. Ann Surg 221(1): 2942, 1995.

17 Dindo D, Demartines N and Clavien PA: Classification of surgical complications: A new proposal with evaluation in a cohort of 6336 patients and results of a survey. Ann Surg 240(2): 205-213, 2004.

18 Saxena A, Yan TD, Chua TC and Morris DL: Critical assessment of risk factors for complications after cytoreductive surgery and perioperative intraperitoneal chemotherapy for pseudomyxoma peritonei. Ann Surg Oncol 17(5): 1291-1301, 2010.

19 Saxena A, Yan TD and Morris DL: A critical evaluation of risk factors for complications after cytoreductive surgery and perioperative intraperitoneal chemotherapy for colorectal peritoneal carcinomatosis. World J Surg 34(1): 70-78, 2010.

20 Elias D, Glehen O, Pocard M, Quenet F, Goere D, Arvieux C, Rat P and Gilly F: A comparative study of complete cytoreductive surgery plus intraperitoneal chemotherapy to treat peritoneal dissemination from colon, rectum, small bowel, and nonpseudomyxoma appendix. Ann Surg 251(5): 896-901, 2010.

21 Howe JR, Karnell LH, Menck HR and Scott-Conner C: The american college of surgeons commission on cancer and the American Cancer Society. Adenocarcinoma of the small bowel: Review of the National Cancer Data Base, 1985-1995. Cancer 86(12): 2693-2706, 1999.

22 Gibson MK, Holcroft CA, Kvols LK and Haller D: Phase II study of 5-fluorouracil, doxorubicin, and mitomycin $c$ for metastatic small bowel adenocarcinoma. Oncologist 10(2): 132-137, 2005.

23 Fishman PN, Pond GR, Moore MJ, Oza A, Burkes RL, Siu LL, Feld R, Gallinger S, Greig P and Knox JJ: Natural history and chemotherapy effectiveness for advanced adenocarcinoma of the small bowel: A retrospective review of 113 cases. Am J Clin Oncol 29(3): 225-231, 2006.

Received November 22, 2016

Revised July 26, 2017

Accepted August 1, 2017 